\title{
UTILIZAÇÃO DE MOTOR DE JOGO PARA TREINAMENTO DE EVACUAÇÃO DE EDIFÍCIOS EM CASO DE INCÊNDIO
}

\author{
Rosana Debiasi ${ }^{1}$ \\ João Carlos Souza ${ }^{2}$
}

\section{RESUMO}

Este artigo tem como objetivo analisar alguns estudos com relação aos jogos sérios e a simulação com a utilização de motor de jogo para a evacuação de edificações em caso de incêndio a partir da revisão de literatura. A evacuação de edifícios em casos de incêndio é um desafio em qualquer circunstância. Para sobreviver a um incêndio, os ocupantes da edificação devem ser capazes de se evadir antes que a situação se torne insustentável. O tempo de esvaziamento do prédio é, portanto, um fator crítico a ser considerado e a falta de conhecimento sobre as noções básicas de segurança contra incêndios pode aumentar perigosamente esse tempo. Nesse sentido, pesquisadores propõem a utilização de jogos sérios, que são aqueles que têm como objetivo principal o aprendizado, e de simulação, como ferramenta para treinamento, planejamento e avaliação de planos de emergência. A partir da utilização de motor de jogo, é possível simular o comportamento de uma multidão de forma rápida. Dentre as vantagens da utilização de motor de jogo, destaca-se a inserção do usuário em um ambiente de realidade virtual em tempo real com vista em três dimensões, e da possibilidade de serem utilizados em diversos dispositivos como computador, console, celular, óculos de realidade virtual (VR).

Palavras-chave: Evacuação de emergência. Jogos sérios. Realidade virtual. Motor de jogo.

\footnotetext{
${ }^{1}$ Aluna do programa de pós-graduação em arquitetura e urbanismo (pós arq). Mestre em engenharia civil pela Universidade Federal de Santa Catarina. E-mail: rosana.debiasi@ufsc.br ${ }^{2}$ Professor efetivo da Universidade Federal de Santa Catarina - UFSC. Doutor em Engenharia de Produção pela Universidade Federal de Santa Catarina. E-mail: joao.carlos@ufsc.br.
} 


\title{
USE OF GAME ENGINE FOR BUILDING EVACUATION TRAINING IN CASE OF FIRE
}

\begin{abstract}
The objective of this article is to analyze some studies regarding serious games and simulation for evacuation of buildings in case of fire with the use of game engine from a literature review. Evacuation of buildings in cases of fire is a challenge under any circumstances. To survive a fire, occupants of the building must be able to evacuate before the situation becomes unsustainable. Therefore, building evacuation time is a critical factor to consider, besides, the lack of knowledge about the basics of fire safety can dangerously increase the evacuation time. In this case, researchers propose the use of serious games, games whose main purpose is education, and simulation as a tool for training, planning and evaluation of emergency plans. Using a game engine, it is possible to create this games and crowd behavior simulations quickly. Some advantages of using a game engine are the user's insertion into a real time virtual reality environment with three dimensional view, and the possibility of being used in various devices such as computer, console, mobile phone, virtual reality glasses (VR).
\end{abstract}

Keywords: Emergency evacuation. Serious games. Virtual reality. Game engine. 


\subsection{INTRODUÇÃO}

O fogo descontrolado é considerado um dos elementos mais perigosos para os ocupantes de uma edificação. Além dos altos custos em danos materiais, são os efeitos sobre os habitantes que têm o maior impacto (ALMEIDA et al., 2011). Alguns exemplos de situações com um grande número de vítimas em função do fogo incluem o incêndio na discoteca Kiss em Santa Maria (Brasil) do qual resultaram em 230 mortes e 160 feridos, no prédio Joelma em São Paulo (Brasil) com 179 mortos e 320 feridos, na ópera de Rhoad em Boyertown (Estados Unidos) com 170 mortos, no hotel Monte Carlo em Las Vegas (Estados Unidos) com 85 mortos e 770 feridos. Tais ocorrências têm como fator agravante a falta de informação e treinamento dos usuários (SILVA et al., 2013a).

Entender como funciona a saída dos usuários em edificações ao enfrentar situações de emergência é uma grande preocupação no meio acadêmico, na comunidade externa, e para profissionais como engenheiros, arquitetos, bombeiros. O comportamento dos usuários ao enfrentar a necessidade de sair rapidamente de um edifício, para uma situação de emergência, seja um incêndio ou algum outro tipo de fatalidade, é um campo específico de pesquisa que vem ganhando muita atenção (HELBING et al., 2002 apud SCHRECKENBERG, M., SHARMA, S, 2002; CORDEIRO et al., 2011a; ALMEIDA et al., 2011).

Uma maneira de minimizar o desespero das pessoas em situações de incêndio são os treinamentos realizados no local. No entanto, esses treinamentos dificilmente são capazes de recriar as verdadeiras condições de pânico e as pessoas tendem a não considerar esse tipo de situação. Além disso, para essa ação é necessário envolver boa parte dos funcionários ou usuários do local (o que nem sempre é possível), bem como causar a 
interrupção das atividades de rotina. Como exemplo, Silva et al. (2013b) verificaram que mesmo sendo bem planejado, o treinamento de evacuação de um hospital pode não impor todo o estresse emocional necessário aos participantes.

Outra alternativa válida é a simulação computacional considerando o comportamento de uma multidão de pessoas em situação de evacuação. No entanto, há situações que dificilmente podem ser reproduzidas devido a sua complexidade e excepcionalidade. Além disso, pode haver limitações com relação ao software de simulação, conforme exposto em um estudo feito por Chiu et al. (2018).

Alguns pesquisadores propuseram a utilização de jogos de computador para treinar e também verificar o comportamento humano (CHITTARO e RANON, 2009; SMITH e TRENHOLME, 2009; KOBES et al., 2009; CORDEIRO et al., 2011b). Esse tipo de jogo, no qual o objetivo principal é a conscientização sobre certos problemas, ensino, educação, entre outras aplicações possíveis, e não o entretenimento, são conhecidos como jogos sérios (SUSI et al., 2007). Esses incorporam a vantagem dos jogos de computador que mantém os jogadores focados, além de incentivá-los a se tornarem espertos na resolução de desafios e melhorar as suas habilidades (CHEN e MICHAEL, 2005).

O termo "jogos sérios" foi introduzido por Clark C. Abt na década de 1970, tendo sido usado pela área militar desde então para treinar habilidades estratégicas por um longo período, chamando-os de "jogos de guerra". O primeiro conjunto de regras para esse tipo de jogo foi criado em 1812 e nomeado "Instruções para a Representação de Manobras Táticas" sob o disfarce de um jogo de guerra. Foi originalmente produzido e desenvolvido pelo tenente Georg Leopold von Reiswitz e seu filho Georg Heinrich Rudolf von Reiswitz do exército prussiano (KRIEGSSPIEL, 2018). 
A partir do motor de jogo é possível fazer esses jogos de maneira rápida e com pouco custo. Isso é possível porque o motor de jogo é um software desenvolvido para fazer jogos sem precisar recodificar elementos como: 1) linguagens de script; 2) controles; 3) inteligência artificial ${ }^{3}$ (Al); e 4) física (COWAN e KAPRALOS, 2014; ESSAYS, UK, 2018; UNITY, 2019). Algumas vantagens de se utilizar o motor de jogo incluem: 1) renderização do que está ocorrendo em tempo real em vista 3D; 2) física (elementos como gravidade e colisão já estão embutidos no motor de jogo); 3) podem ser jogados por diversos usuários em rede interna (intranet) ou externa (internet); 4) podem ser utilizados em diversos dispositivos, como computador, console, celular, óculos de realidade virtual (VR); 5) possibilidade de importar arte, recursos 2D e 3D de outros aplicativos; e 6) possibilidade de acréscimo de personagens com Inteligência artificial (KIRNER e SISCOUTO, 2007; LEWIS e JACOBSON, 2002; TRENHOLME e SMIDTH, 2008; UNITY, 2019a). Ressalta-se que existem atualmente diversos motores de jogo gratuitos que podem ser utilizados para a criação de jogos, tais como: Unity, Unreal, Source, Blender, dentre outros (BLENDER, 2019; SMITH e TRENHOLME, 2009; SOURCE, 2019; UNITY, 2019b; UNREAL, 2019).

Além disso, foram verificados em estudos que é possível, através do motor de jogo, fazer a simulação de evacuação de edifícios (CHIU et al., 2018; MÓL et al., 2011; XI e SMIDTH, 2015) e até mesmo juntar elementos de simulação de evacuação com o conceito de jogos sérios (ASANO et al., 2014).

Baseado na importância de se estudar como ocorrem evacuações de incêndio nas edificações e das vantagens verificadas ao se utilizar um motor de jogo, será feito neste artigo a análise de alguns estudos com relação aos "jogos sérios" e a "simulação" a partir da utilização de motor de jogo. Esses estudos foram selecionados a partir de uma pesquisa bibliográfica, utilizando

\footnotetext{
${ }^{3}$ Inteligência artificial é uma área de pesquisa da ciência da computação dedicada a simular processos e ações de pensamento como perseguir, desviar e fugir em combinação com uma forma para encontrar o caminho a partir de um trajeto mais curto entre dois pontos (pathfinding).
} 
ferramentas de busca como a base de dados Science Direct, IEE Explore e Google acadêmico. Foram pesquisados artigos que abordam os assuntos "jogos sérios" (serious games), "simulação", "evacuação de incêndios" (fire evacuation) e "motor de jogo" (game engine). A seguir, no item 2.0 e 3.0 serão apresentados alguns estudos relacionados, respectivamente, a "jogos sérios e motor de jogo", e "simulação e motor de jogo".

\subsection{JOGOS SÉRIOS E MOTOR DE JOGO}

Almeida et al. (2017) fizeram um experimento a fim de investigar o comportamento humano em situações de evacuação de incêndio a partir de um jogo sério. Para isso, foi feito um jogo 3D em primeira pessoa (refere-se à perspectiva gráfica que segue do ponto de vista do personagem controlado pelo jogador, como se o personagem do jogo e o jogador fossem o mesmo observador) com o motor de jogo Unity que funcionou da seguinte forma: a pessoa está dentro de uma sala de trabalho em frente a um corredor com duas saídas possíveis: direita e esquerda. Ambas as saídas têm o mesmo comprimento para uma porta de saída e são completamente simétricas. Neste local há 5 cenários: 1) não há indicação com placa de saída da edificação; 2) há indicação com placa de saída da edificação (para a esquerda); 3) igual ao segundo cenário, mas ao lado esquerdo do corredor há fumaça; 4) igual ao segundo cenário, mas ao lado esquerdo do corredor há fumaça e fogo; e 5) igual ao segundo cenário, mas as pessoas estão correndo pelo sentido contrário (direita). O voluntário responde a um questionário acerca de que direção escolheria para sair se fossem apresentados em cada cenário (se pela esquerda ou direita) e depois joga nesses locais.

Para validar o experimento, um grupo de 16 especialistas em segurança contra incêndio, planejamento de emergência e evacuação de edifícios foram consultados. Eles responderam a um questionário para avaliar a experiência do 
jogo e também validaram as perguntas a serem apresentadas no questionário. Após isso, foi selecionado um grupo de 62 pessoas, com idade entre 12 e 76 anos, $54,8 \%$ do sexo masculino e $45,2 \%$ do sexo feminino. Grande parte dos voluntários afirmou ter experiência com tecnologia da informação (95,2\%), $46,8 \%$ dos voluntários declararam jogar vídeo games frequentemente e 25,8\% possuem treinamento em segurança contra incêndio, conforme mostrado na Tabela 01.

Tabela 01: Perfil dos voluntários

\begin{tabular}{l|l}
\hline Dado & Valor \\
\hline Número de voluntários (total) & $62(100 \%)$ \\
\hline Homens & $34(54,8 \%)$ \\
\hline Mulheres & $28(45,2 \%)$ \\
\hline Idade mínima & 12 \\
\hline Idade máxima & 76 \\
\hline Experiência com tecnologia de informação & $59(95,2 \%) \operatorname{sim} / 3(4,8 \%)$ não \\
\hline Experiência em vídeo games & $29(46,8 \%) \operatorname{sim} / 33(53,2 \%)$ não \\
\hline Treinamento em segurança contra incêndio & $16(25,8 \%) \operatorname{sim} / 46(74,2 \%)$ não \\
\hline
\end{tabular}

Fonte: Almeida et al., 2017 (adaptado)

Os resultados mostram que no cenário 1 , quando não há a placa de saída os voluntários tendem a sair pela direita, no cenário 2, todos os voluntários seguiram a indicação de placa da saída (esquerda), no cenário 3 , com fumaça do lado esquerdo, quase a metade (48\%) resolveu arriscar e sair pela esquerda, no cenário 4 , com fogo na esquerda, todos optaram pela direita, e no cenário 5 mais da metade (65\%) foi influenciada pelo grupo a frente que já estava correndo e utilizando a saída pela direita, conforme apresentado na Figura 01. Com relação às respostas dadas pelos voluntários no questionário e depois ao jogar, observou-se uma pequena diferença entre os cenários 1 , 3 e 5 , sendo maior no 5 o que mostra o efeito de influência do grupo que está a frente (Tabela 02). Observou-se que em ambos os experimentos (questionário e jogos sérios) a maioria dos usuários optou pela resposta correta e a 
Revista Científica do Corpo de Bombeiros Militar de Pernambuco

Artigo Publicado no Vol.05 N.13 - Edição Jul a Dez 2019 - ISSN 2359-4829

Versão on-line disponível em: http://www.revistaflammae.com

sinalização do local de saída (cenário 2) teve uma grande influência na escolha da rota de fuga.

Figura 01: Cenários e escolha do sentido de saída (esquerda ou direita) dos voluntários

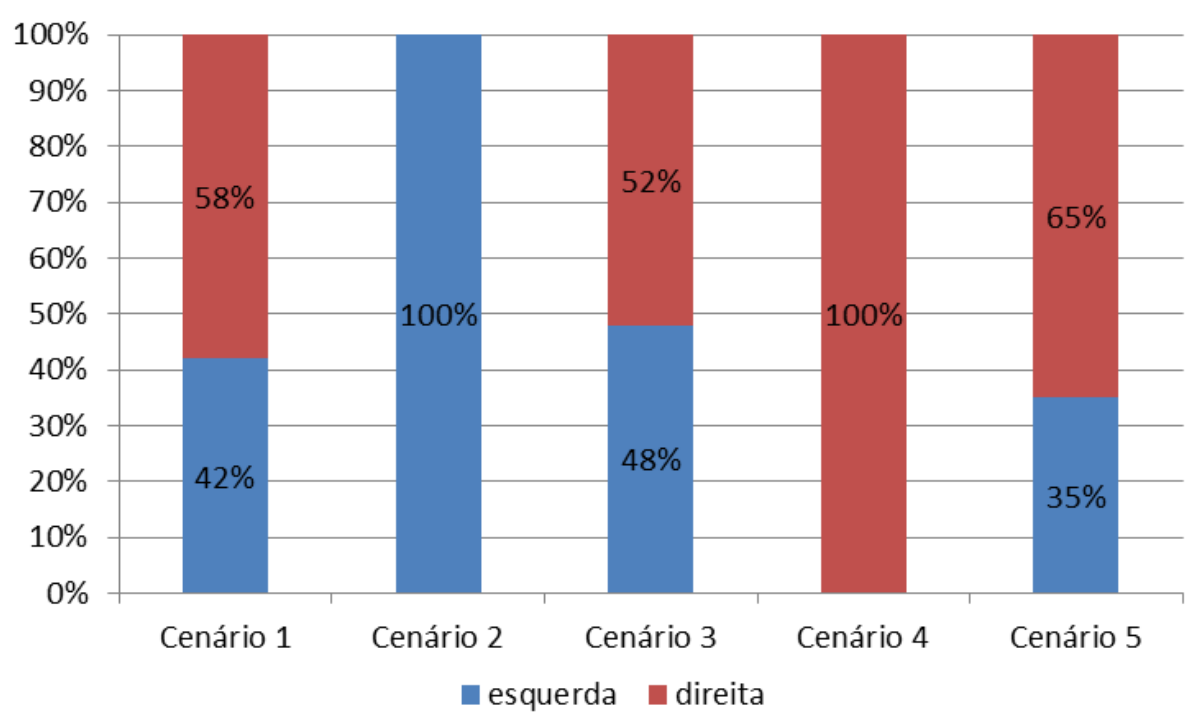

Fonte: Almeida et al., 2017 (adaptado)

Tabela 02: Respostas dos voluntários com relação aos questionários e ao jogo

\begin{tabular}{c|c|c|c|c}
\hline \multirow{2}{*}{ Cenário } & \multicolumn{2}{|c|}{ Questionário } & \multicolumn{2}{c}{ Jogo Sério } \\
\cline { 2 - 5 } & Esquerda & Direita & Esquerda & Direita \\
\hline 1 & 21 & 41 & 26 & 36 \\
\hline 2 & 62 & 0 & 62 & 0 \\
\hline 3 & 18 & 44 & 30 & 32 \\
\hline 4 & 0 & 62 & 0 & 62 \\
\hline 5 & 16 & 46 & 22 & 40 \\
\hline
\end{tabular}

Fonte: Almeida et al., 2017 (adaptado)

Silva et al. (2013b) fizeram um jogo sério chamado de "EVA" (abreviação de evacuação) para analisar a fuga de usuários em um prédio de hospital. Foi feito um modelo 3D da edificação no software Revit, o qual foi importado para o motor de jogo Unity. O jogo é em 3D, em primeira pessoa. Foram selecionados 20 voluntários para o experimento. A idade média do grupo é de 24 anos, com 
$45 \%$ de homens e $55 \%$ de mulheres. Além disso, $65 \%$ do grupo são jogadores frequentes de jogos de computador. O objetivo do jogo é a evacuação de uma sala de hospital no momento em que é tocado o alarme de incêndio.

O jogador inicia em um corredor (logo após sair do elevador) empurrando a cadeira de rodas em que está o senhor Adam em direção a sua sala. Em seguida, o alarme de incêndio toca. No momento em que isso ocorre é apresentado um questionário com a seguinte pergunta "O alarme de incêndio toca. O que você deve fazer?" após é necessário escolher uma das seguintes opções: 1) "Nada. Provavelmente é um falso alarme"; 2) "Esperar. Se for um alarme real, o pessoal da segurança dará um alerta"; 3) "Tentar entender o que está havendo"; e 4) "Sair da edificação o mais rápido possível". Após o participante responder, independente da opção escolhida, no jogo é mostrado que é necessário sair da edificação o mais rápido possível. Se o voluntário optar por levar Adam, no jogo é apresentado que foi feita uma boa ação. Além disso, caso o participante quisesse utilizar o elevador, no momento em que o mesmo aperta o botão do elevador é mostrada a mensagem "Em caso de incêndio, não use o elevador. Utilize as escadas para a saída". O jogo termina quando o voluntário acha uma das 4 saídas válidas e sai da edificação. Ressalta-se que o jogador pode optar ou não deixar Adam em uma área segura e escolher uma das 4 saídas da edificação (duas de emergência, uma na entrada principal e outra nos fundos da edificação). Após o término do jogo, o jogador responde um questionário que inclui perguntas como: nome, sexo, idade, ocupação, se já esteve envolvido em uma situação de incêndio na vida real, se teve treinamento anterior em evacuações de emergência, e se já participou de exercícios de incêndio.

Observou-se no estudo que o tempo de evacuação médio é de 84,6 segundos, que houve 2 pessoas que levaram mais tempo para evacuação (entre 240 e 270 segundos) por não estarem familiarizados com jogos, e esse tempo de evacuação pode chegar até 63,6 segundos para quem já é familiarizado com jogos. Com relação ao questionário apresentado no início do 
jogo, 18 pessoas escolheram a opção 3 (tentar entender o que está acontecendo) ou 4 (sair da edificação o mais rápido possível), com 9 pessoas em cada, e 2 pessoas selecionaram a alternativa 1 (não fazer nada, provavelmente é um alarme falso) ou 2 (esperar pelas instruções da segurança), com um indivíduo cada. Ressalta-se que as opções escolhidas pela maioria dos voluntários (3 ou 4) são válidas em uma situação real. Além disso, a maioria dos voluntários (17 pessoas) optou por levar Adam a uma área segura.

Ao comparar as respostas dos questionários e o que os voluntários fizeram no jogo somente duas pessoas responderam diferente do que fizeram no jogo: 1 escolheu a opção 1 (é um falso alarme) e outro a opção 2 (esperar). Como a amostra foi pequena (20 pessoas) os autores comentam que os próximos passos da pesquisa sejam feitos com mais pessoas. Além disso, foi apontado pelos voluntários que o jogo serve como um treinamento para situações de emergência e o uso de joystick poderia superar problemas que alguns jogadores experimentaram ao utilizar simultaneamente o teclado e 0 mouse.

Uma abordagem semelhante é feita por Chittaro e Ranon (2009). Foi feito um jogo sério em 3D, em primeira pessoa para um edifício universitário no motor de jogo NeoAxis. Foram selecionadas 7 pessoas que trabalham há pelo menos 2 anos na edificação por se tratar de um estudo preliminar. Ambos são familiarizados com jogos 3D. Basicamente o jogo reproduz uma edificação de 3 andares com 100 salas; há fenômenos e objetos diretamente relacionados com emergência e evacuação de incêndio, como: fogo, fumaça, saídas de emergência, alarmes de incêndio, inclusive alarmes sonoros; e há objetos comuns nos escritórios e laboratórios como computador, telefone, documentos, malas, chaves do carro com os quais o jogador pode interagir.

O voluntário joga em diferentes cenários, iniciando com grau de dificuldade menor até chegar ao maior. Se o usuário realizar as ações 
necessárias para evacuação, ele passará para o próximo cenário. Foram feitas 3 hipóteses: 1) um pequeno incêndio no computador próximo ao jogador; 2) fogo e fumaça em um laboratório próximo do escritório em que está o jogador; 3) alarme de aviso toca (som não contínuo) após entrada de fumaça no escritório onde está o jogador e em seguida o alarme de incêndio (som contínuo) é acionado. O jogador deve decidir se leva objetos importantes junto e em seguida evacuar o edifício. No momento em que o voluntário inicia o jogo, é requisitado ao mesmo que escolha o sexo (masculino ou feminino), idade e peso. Essa escolha influenciará a velocidade de corrida e a força do personagem durante o jogo. Após selecionar o personagem, o jogador inicia no cenário 1 (mais fácil). Ao chegar ao final de cada cenário (sair da edificação), é reportado pelo jogo sobre o sucesso ou fracasso, o tempo necessário para jogá-lo e as ações certas e erradas, explicando também por que foi considerado certo ou não.

A partir desses dados é calculada a pontuação, que serve também para o jogador comparar como foi em diferentes partidas ao jogar o mesmo cenário. Os resultados do jogo sério foram integrados com a ferramenta VUflow (CHITARO et al., 2006) e apresentados em um mapa 2D com: visualizações não agregadas que mostram os caminhos percorridos por cada jogador nos cenários, e visualizações agregadas com o objetivo de destacar padrões de navegação da população (por exemplo, identificando áreas viajadas no ambiente virtual). Esses resultados permitem analisar o comportamento dos usuários. Além disso, é possível aprender e praticar numa variedade de situações diferentes algumas das ações e procedimentos necessários em emergências de incêndio.

Smith e Trenholme (2009) modelaram uma edificação universitária no motor de jogo Source, consistindo em um jogo 3D com visão em primeira pessoa. Foram modelados elementos como alarme de incêndio, extintores, saídas de emergência, tal como posicionados na edificação real. A edificação estudada é o Departamento de Ciência da Computação da universidade de 
Durham que possui três andares e é composta por salas de escritórios, salas de encontro e uma recepção no primeiro andar. Há três saídas: a entrada principal no térreo, uma saída nos fundos do primeiro andar, e um corredor de conexão entre o departamento de ciência da computação e o de engenharia do segundo andar. Foram tiradas fotos de texturas da edificação real e as mesmas foram inseridas na modelagem para dar um ar mais realista a simulação do jogo sério.

Três cenários foram criados. Em cada um deles um gatilho é acionado na sala de segurança que está no último andar da edificação. A diferença entre esses locais é onde o fogo inicia. Em cada cenário é acionado um gatilho na sala de segurança que está no último andar da edificação. $O$ jogador inicia em uma sala térrea da edificação e é instruído pelo tutor que acompanha o experimento a ir em uma sala no terceiro andar e esperar 3 segundos. Após esse tempo, o alarme de incêndio é acionado, fechando todas as portas dos escritórios dos corredores, que inicialmente estão abertas. Ao tocar o alarme de incêndio, o jogador é instruído para sair o mais rápido da edificação evitando rotas com fumaça e fogo. Quando o usuário encontra a saída, o jogo é finalizado. Após o jogo, deve ser respondido um questionário com relação à avaliação da: dificuldade de navegação, velocidade de movimento do personagem, realismo da edificação, realismo da tarefa a ser executada, e o foco/atenção proporcionado pelo experimento. Foram selecionados 20 participantes, sendo 2 mulheres e o restante homens. Todos os usuários trabalham diariamente com computadores e sete deles jogam no computador pelo menos uma vez por semana.

Resultados demonstraram que o tempo médio de evacuação da edificação no cenário 1, 2 e 3 foi de, respectivamente, 165,83 s, 66 s e 138,58 segundos. Tempos menores que esses foram obtidos por participantes com experiência em jogar no computador, com tempos no cenário 1, 2 e 3 de, respectivamente, 73 segundos, 34 segundos e 76 segundos, aproximadamente $50 \%$ menor que a média. Apesar disso, ressalta-se que em ambos os 
resultados (de se ter ou não conhecimento em jogos) seguem um mesmo padrão, no qual se leva menos tempo em sair no segundo cenário em comparação com o restante (1 e 3). Com relação às respostas do questionário, a média dos participantes considerou o ambiente da edificação bastante realista, o foco e o realismo da tarefa alto. Foram relatadas dificuldades pelas pessoas sem experiência em jogar computador com relação ao controle e velocidade do personagem. Além disso, foi possível traçar o comportamento de casa usuário no teste. Os autores pretendem testar futuramente esse jogo em um ambiente de multijogadores (multiplayer) e aumentar o número de participantes.

\subsection{SIMULAÇÃO E MOTOR DE JOGO}

Mól et al. (2011) utilizaram o motor de jogo Unreal para simular o tempo de evacuação em uma edificação e comparar com o tempo utilizado por pessoas no mesmo percurso. O local escolhido foi a universidade Gama Filho. A edificação foi modelada e passada para o motor de jogo Unreal. Foram modelados avatares autônomos (ou bots) e os caminhos de evacuação sinalizados através de nós (node), observando os caminhos principais e secundários. Os avatares autônomos são personagens não jogáveis com inteligência artificial (Al) capazes de se locomoverem por conta própria (sem necessidade de recrutar voluntários para controlá-los) seguindo regras especificadas pelo programa. Seis rotas de saída foram definidas para análise comparativa entre o real e o simulado (Tabela 03). Observou-se na Tabela 03 uma boa concordância entre os tempos reais e simulados durante os experimentos de evacuação em todas as rotas. Esses resultados validam a utilização do motor de jogo Unreal para simulação de evacuação em situações de emergência. Além disso, os autores observam que com a simulação a partir do uso de avatares autônomos diminui-se a necessidade do recrutamento de 
Revista Científica do Corpo de Bombeiros Militar de Pernambuco

Artigo Publicado no Vol.05 N.13 - Edição Jul a Dez 2019 - ISSN 2359-4829

Versão on-line disponível em: http://www.revistaflammae.com

usuários para participar de simulações em rede (mais de um usuário jogando no mesmo ambiente em realidade virtual).

Tabela 03: Comparação entre os tempos de evacuação

\begin{tabular}{c|c|c}
\hline \multirow{2}{*}{ Rota } & \multicolumn{2}{|c}{ Tempo de evacuação (minutos: segundos: centésimos) } \\
\cline { 2 - 3 } & Em experimentos reais & Simulação com a Unreal \\
\hline Rota 1 & $01: 15: 04$ & $01: 14: 19$ \\
\hline Rota 2 & $01: 01: 27$ & $00: 57: 18$ \\
\hline Rota 3 & $01: 33: 48$ & $01: 32: 35$ \\
\hline Rota 4 & $01: 55: 52$ & $01: 54: 21$ \\
\hline Rota 5 & $01: 38: 90$ & $01: 42: 32$ \\
\hline Rota 6 & $01: 55: 65$ & $01: 58: 61$ \\
\hline
\end{tabular}

Fonte: Mól et al., 2011 (adaptado)

Chiu et al. (2018) examinaram a aplicabilidade de pacotes do software EXODUS e do motor de jogo Unity para simular o tempo necessário para evacuação em situação de incêndio dos avatares autônomos sob condições idênticas. Para validar os resultados, foi feita uma simulação em espaço real em uma sala de $100 \mathrm{~m}^{2}$ com 80 pessoas e a saída com abertura de $1 \mathrm{~m}$. Os autores ressaltaram que para fazer a simulação no software EXODUS é necessário utilizar um grid de 0,5 m x 0,5 m como módulo de mobilidade dos avatares autônomos. Já no motor de jogo Unity é utilizado o algoritmo NavMesh, que indica a posição do ocupante por triangulação, o que torna mais precisa a mobilidade do mesmo. Observou-se que os resultados apresentados pela Unity eram os que mais se aproximavam da simulação com o ambiente real.

Já os autores Xi e Smidth (2015) utilizaram resultados da simulação de cada um dos caminhos possíveis para evacuação de incêndio provenientes do software FDS+Evac e o incluíram no motor de jogo Unity. O modelo consiste em um andar com 5 salas e três saídas válidas. Foram comparados os tempos de evacuação entre: somente o software FDS+Evac, e a combinação entre o software FDS+Evac e o motor de jogo Unity. Verificou-se pouca diferença com 
relação aos tempos médios de evacuação entre a Unity e o FDS+Evac. A diferença se deu devido à colisão existente em cada programa. Os autores observaram que a colisão no motor de jogo Unity é detectada com maior precisão que no software Evac.

Asano et al. (2014) desenvolveram um sistema multiagente no motor de jogo Unity que permite simular em três dimensões o comportamento de diversos passageiros (avatares autônomos) de um aeroporto em um jogo de computador. Os avatares autônomos dinamicamente checam os arredores por sinais visíveis utilizando algoritmos de percepção 3D. Cada sinal é anotado com a direção de uma ou mais saídas. Se um sinal for percebido corretamente, o avatar autônomo interpretará sua informação direcional em relação ao seu próprio local. Os autores observaram que os resultados das simulações são bastante similares com o comportamento real dos usuários (visto por câmeras do aeroporto). Além disso, observou-se que esse experimento pode ser combinado com o "Oculus Riff" em algum voluntário e ser testado no mesmo terminal do aeroporto virtual como um jogo sério. Portanto, é possível utilizar a simulação aliada ao jogo sério em um motor de jogo.

\subsection{CONSIDERAÇÕES FINAIS E CONCLUSÕES}

A partir dos estudos descritos, conclui-se que os jogos sérios podem ser utilizados para a complementação da formação e treino em situações de emergência com incêndios, além de ser possível recolher o comportamento de cada usuário ou da população em análise. Os jogos sérios também se mostraram como uma alternativa para treinamento dos usuários em situações extremas (fogo, explosão, por exemplo), o que não seria possível com o treinamento de incêndio in loco. Além disso, a experiência dos voluntários (com conhecimento prévio ou não em jogos de computador ou vídeo game) ao jogar em jogos sérios, no qual está inserido em um ambiente de realidade virtual, 
permite que haja sugestões com relação a melhorias do sistema de evacuação da edificação.

Nota-se que os resultados de simulação de evacuação em motores de jogo como Unreal e Unity são semelhantes ao que é visto na vida real. Verificase que em motores de jogo, como a Unity, possuem um algoritmo mais complexo para o deslocamento de avatares autônomos, com triangulação em três dimensões, enquanto que em um software de simulação como o EXODUS o deslocamento dos mesmos é feito a partir de uma malha, sendo, portanto, menos precisos os resultados. Além disso, observa-se que a utilização de avatares autônomos melhora o desempenho da simulação em situações de multidão. Ainda se verifica que na simulação a partir da utilização de motores de jogo é possível a adição de realidade virtual com o conceito de jogos sérios.

Em geral, percebe-se que o motor de jogo possui diversas vantagens para se utilizar em eventos com realidade virtual no conceito de jogos sérios, para a simulação de evacuação de multidões em caso de incêndio, bem como para essas duas vertentes juntas. Em todas elas é possível aprender com relação ao comportamento dos usuários na evacuação de incêndio, além de permitir verificar com mais facilidade melhorias a serem feitas no sistema de prevenção de incêndio.

Ressalta-se que a experiência dos usuários com jogos sérios, e/ou as simulações do comportamento de multidão a partir do uso de motor de jogo não substitui o treinamento de incêndio no local e não é o suficiente para preparar uma pessoa para reagir a emergências. No entanto, essas ferramentas, conforme apresentados em estudos nesse trabalho, podem auxiliar os projetistas para programar e realizar melhorias no sistema preventivo de incêndio. 


\subsection{AGRADECIMENTOS}

Ao professor Dr. João Carlos Souza pela disciplina ministrada no programa de pós graduação em arquitetura (pós arq) na Universidade Federal de Santa Catarina (UFSC) que possibilitou a confecção deste artigo, e ao servidor Ricardo César dos Passos, diretor do departamento de Gestão da Informação (DGI) da UFSC, pela liberação dada para fazer essa disciplina.

\subsection{REFERÊNCIAS BIBLIOGRÁFICAS}

ABT, C., Serious Games. Vicking Press, 1970.

ALMEIDA et al. Serious games for the human behaviour analysis in emergency evacuation scenarios. Cluster Comput, v. 20, p. 707-720, 2017.

ALMEIDA et al. Crowd simulation modeling applied to emergency and evacuation simulations using multi-agent systems. In: SOUSA, A., OLIVEIRA, E. (eds.) DSIE11- 6th Doctoral Symposium on Informatics Engineering, p. 93-104. Faculdade de Engenharia da Universidade do Porto, 2011.

ASANO et al. A Multi-agent System based on Unity 4 for Virtual Perception and Wayfinding. Transportation Research Procedia, v.2, p. 452-455, 2014.

BLENDER. Blender. Disponível em: https://www.blender.org/ Acesso em junho de 2019.

CHIU et al. Study on evacuation simulation under crowd-diversion condition. Advances in Mechanical Engineering, v. 10, n.7, p. 1-8, 2018.

CHITTARO et al. VU-Flow: A Visualization Tool for Analyzing Navigation in Virtual Environments. IEEE Transactions on Visualization and Computer Graphics, v; 12, p. 1475- 1485, 2006.

CHITTARO, L.; RANON, R. Serious games for training occupants of a building in personal fire safety skills. In: 2009 Conference in Games and Virtual Worlds for Serious Applications. p. 76-83, 2009. 
CORDEIRO et al. Fire and Evacuation Modeling Technical Conference, Baltimore, Marilândia, 15 a 16 de agosto de 2011, 2011 a.

CORDEIRO et al. Human behavior under fire situations: a case study in the Portuguese Society. In: Advanced Research Workshop: Evacuation and Human Behavior in Emergency Situations (GIDAI, pp. 63-80. Universidade Cantabria, Santander, 2011b.

COWAN, B. KAPRALOS, B. A Survey of Frameworks and Game Engines for Serious Game Development. in: 2014 IEEE 14th International Conference on Advanced Learning Technologies. Atenas, 2014. p. 662-664.

ESSAYS, UK. (Novembro de 2018). Concepts and Features of Game Engines. Disponível em: https://www.ukessays.com/essays/computerscience/concepts-features-game-engines-6438.php?vref=1 Acesso em maio de 2019.

HELBING et al. Simulation of pedestrian crowds in normal and evacuation situations, In: SCHRECKENBERG, M., SHARMA, S. Pedestrian and Evacuation Dynamics, v. 21, p. 21-58. Springer, Berlim, 2002.

KIRNER, C., SISCOUTTO, R. (editores) Realidade Virtual e Aumentada: Conceitos, Projeto e Aplicações. Editora SBC - Sociedade Brasileira de Computação, Porto Alegre, 2007. "Livro do pré-simpósio, IX Symposium on Virtual and Augmented Reality".

KOBES et al. Serious gaming for behavioural assessment and research in case of emergency. An evaluation of experiments in virtual reality. In: SimTecT 2009 Simulation-Concepts, Capability and Technology, Adelaide, 2009.

KRIEGSSPIEL, Origens do Kriegsspiel (online). Em: http://www.kriegsspiel.org.uk/ index.php?option=com content\&task=view\&id=15\&ltemid=58 Acesso em: dezembro de 2018.

LEWIS, M., JACOBSON, J. Introduction, Communications of the Association for Computing Machinery (CACM). Volume especial no Game Engines in Scientific Research, v. 45, p. 27-31, 2002. 
MICHAEL , D.R., CHEN, S. L. Serious Games: Games That Educate, Train, and Inform. Muska \& Lipman/Premier-Trade, 2005.

MÓL et al. Virtual evacuation simulation with autonomous Avatars In: International Nuclear Atlantic Conference - INAC, Belo Horizonte, Brasil, 2011. SILVA, J. et al. Gamifying evacuation drills. In: Iberian Conference on Information Systems and Technologies, CISTI, Lisboa, 2013a.

SILVA, J. et al. A Serious Game for EVAcuation Training In: IEEE 2nd International Conference on Serious Games and Applications for Health (SeGAH), Vilamoura, Portugal, 2013b.

SMITH, S.P., TRENHOLME, D. Rapid prototyping a virtual fire drill environment using computer game technology. Fire Saf. J. v. 44, p. 559-569, 2009.

SOURCE. Source. Valve developer community. Disponível em: https://developer.valvesoftware.com/wiki/Source Acesso em junho de 2019.

SUSI, T. et al. Serious games - An overview. Technical Report HS- IKI -TR07-001, Universidade de Skövde, Suécia, 2007.

TRENHOLME, D., SMIDTH, P. Computer game engines for developing firstperson virtual environments. Virtual Reality v.12, p. 181-187, 2008.

UNITY. Game engines, how do they work? Disponível em: https://unity3d.com/what-is-a-game-engine Acesso em maio de 2019. 2019a.

UNITY. The world's leading real-time creation platform. Disponível em: https://unity3d.com/pt/unity? ga=2.124036682.1364456835.1560350389-

856273433.1560350389 Acesso em junho de 2019. $2019 \mathrm{~b}$.

UNREAL. Make something Unreal. Disponível em: https://www.unrealengine.com/en-US/ Acesso em junho de 2019.

$\mathrm{XI}, \mathrm{M}$;; SMITH, S. Exploring the Reuse of Fire Evacuation Behaviour in Virtual Environment In: Proceedings of the 11th Australasian Conference on Interactive Entertainment (IE 2015), Sidney, Australia, 27 - 30 janeiro de 2015. 